\title{
Optimisation of the synchronisation of a class of chaotic systems: combination of sliding mode and feedback control
}

\author{
Michaux Kountchou* \\ Department of Physics, \\ Laboratory of Electronics and Signal Processing, \\ Faculty of Science, \\ University of Dschang, \\ P.O. Box 67, Dschang, Cameroon \\ and \\ Nuclear Technology Section, \\ Institute of Geological and Mining Research, \\ P.O. Box 4110, Yaounde, Cameroon \\ Email: mkountchounoube@yahoo.fr \\ *Corresponding author

\section{Patrick Louodop} \\ Department of Physics, \\ Laboratory of Electronics and Signal Processing, \\ Faculty of Science, \\ University of Dschang, \\ P.O. Box 67, Dschang, Cameroon \\ Email: louodop@yahoo.fr
}

\section{Samuel Bowong}

Department of Mathematics and Computer Science, Laboratory of Applied Mathematics,

Faculty of Science,

University of Douala,

P.O. Box 24157, Douala, Cameroon

Email: samuelbowong@yahoo.fr

\section{Hilaire Fotsin}

Department of Physics,

Laboratory of Electronics and Signal Processing,

Faculty of Science,

University of Dschang,

P.O. Box 67, Dschang, Cameroon

Email: hbfotsin@yahoo.fr 


\begin{abstract}
This paper addresses the problem of optimisation of the synchronisation for a class of uncertain chaotic systems from a control theoretic point of view. A robust adaptive feedback which accomplishes the synchronisation of chaotic systems using an optimal tuning scheme based on Riccatti equations is successfully adapted. The underlying idea is to optimise the synchronisation of chaotic systems by accounting the control effort despite the uncertainties. The approach developed considers incomplete state measurements and no detailed model of the systems to guarantee robust stability. This approach includes a high-order sliding mode estimator and leads to a robust adaptive feedback control scheme. A finite horizon can be arbitrarily established by ensuring that the chaos synchronisation is achieved at established time. An advantage is that the studied scheme accounts the energy wasted by the controller and the closed-loop performance on synchronisation. Both mathematic proof and numerical simulations are presented to show the feasibility of the optimisation strategy for establishing the synchronisation of chaotic systems even if there are some modelling mismatches and parametric variations.
\end{abstract}

Keywords: chaos synchronisation; optimal feedback control; Ricatti equation.

Reference to this paper should be made as follows: Kountchou, M., Louodop, P., Bowong, S. and Fotsin, H. (2017) 'Optimisation of the synchronisation of a class of chaotic systems: combination of sliding mode and feedback control', Int. J. Nonlinear Dynamics and Control, Vol. 1, No. 1, pp.51-77.

Biographical notes: Michaux Kountchou is a researcher at the Nuclear Technology Section, Institute of Geological and Mining Research, Yaounde, Cameroon. He received his MS in Physics from the University of Dschang, Cameroon, under the advisory of Prof. Hilaire Fotsin in 2010. He is a PhD Student at University of Dschang, under the advisory of Prof. Hilaire Fotsin and Prof. Samuel Bowong. His current research interests include nuclear instrumentation, implementation of the new chaotic circuits, optimal and robust finite-time control and synchronisation of non-linear systems with applications.

Patrick Louodop is an Assistant Lecturer at the Department of Physics, University of Dschang, Cameroon. He received his $\mathrm{MS}$ and $\mathrm{PhD}$ in Physics from the University of Dschang, Cameroon, under the advisory of Prof. Hilaire Fotsin. His current research interests include finite-time and robust control and synchronisation of non-linear systems with applications.

Samuel Bowong received his PhD in Applied Mathematics from the University of Metz, France in 2003 and MS in Control Theory from the University of Abomey Calavi, Benin in 2000. In 2005, he became a Lecturer and in 2006, a Senior Lecturer. Currently, he is an Associate Professor at the Department of Mathematics and Computer Science, Faculty of Science, University of Douala, where he currently teaches and performs research. His current research interests include non-linear control theory, robust control with applications and mathematical epidemiology.

Hilaire Fotsin is a Leader of Laboratory of Electronics and Signal Processing, Department of Physics, Faculty of Science, University of Dschang, Cameroon. $\mathrm{He}$ is an Associate Professor at the Department of Physics, University of Dschang, Cameroon, where he currently teaches and performs research. His current research interests include nonlinear dynamics and chaos, chaos control and synchronisation in electronics, FPGA. 


\section{Introduction}

Since the idea of synchronising chaotic systems was introduced by Pecora and Carroll (1990), chaos synchronisation has received increasing attention due to its theoretical challenge and its great potential applications in secure communication (Murali and Lakshmanan, 1998), chemical reaction and biological systems (Chen and Dong, 1998; Mao et al., 2009) and so on. Due to the wide potential applications of chaos synchronisation, various synchronisation schemes have been proposed in the last two decades both in theoretical analysis and experimental implementations, such as generalised synchronisation (Murali and Lakshmanan, 1998; Yang and Duan, 1998; Wang and Guan, 2006), phase synchronisation (Michael et al., 1996; Santoboni et al., 2001), lag synchronisation (Taherion et al., 1999; Chen et al., 2007), anti-synchronisation (Li, 2005; Hu et al., 2005; Li and Zhou, 2006) and so on. But despite the amount of theoretical and experimental results already obtained, chaos synchronisation seems difficult task, over all if we think that:

1 due to sensitive dependence of chaos on initial conditions, it is almost impossible to reduce the same starting conditions

2 in matching exactly the master and slave systems, even infinitesimal parametric variations of any model will eventually result in divergence of orbits starting nearby each other

3 parametric differences between chaotic systems (for instance, due to inaccuracy design or time variations) yield different attractors.

However, in practice, due to the inconsistence of the operational conditions (such as the environment, the load, the operational frequency), system uncertainties are unavoidably existed; i.e., exact knowledge of the system dynamics is not possible. A more realistic situation is to know some nominal functions of the corresponding nonlinearities, which are employed in the control design. However, the use of nominal model nonlinearities can lead to performance degradation and even closed-loop instability. In fact, when the systems possess strong nonlinearities, the standard linearising, generic model, and active controllers cannot cancel completely such nonlinearities, and instabilities can be induced. The worst case occurs if knowledge of the nonlinearities is very poor or completely absent, such that conventional linearising techniques are inadequate. Robust synchronisation, however, may provide a possible candidate to deal with this problem, see examples (Han, 1995; Femat et al., 1999; Femat and Solís-Perales, 2008), and references therein. In particular, several authors have reported adaptively estimation techniques. The proposed techniques present an acceptable performance and allow synchronisation, although the parameters are not known (Kammogne and Fotsin, 2014; Mbe et al., 2014) or they are time-varying. But the only drawback of these strategies is that the structure of parameters for a given model must be known. Although the structure of the parameters can be known in some cases, it would be desirable to have a scheme to achieve synchronisation even if slave oscillator has little prior knowledge about the master system. This necessity of robustness can be required in some systems (for instance, the multimode laser, animal gait or oscillatory neural systems). 
The geometric approach for the design of nonlinear controllers based on uncertainty observers has been employed, and these kinds of techniques show satisfactory capabilities for a wide range of systems (Aguilar et al., 2002; Aguilar-Lopez and Alvarez-Ramirez, 2002). The use of proportional observers coupled with linearising controllers has been very successful, but the proportional observers have several problems. Notably, they are very sensitive to noisy measurements, and robustness issues have not been completely addressed. For these reasons, more sophisticated observers have been designed, in order to generate better open-loop and closed-loop performances. For some, a high-order sliding mode observer has been designed to reconstruct the dynamics of the output error and its time derivatives. Then, based on the reconstructed information, a output-feedback control signal is constructed to force the slave converge to the master. This scheme does not require the priori information about the system model to carry out the chaos suppression (or synchronisation) and only one controller parameter is required to tune, thus making the complex control problem become physically realisable.

However, despite the large amount of theoretical and experimental results already obtained, a great deal of effort is still required to determine the optimal parameters, in order to shorten the synchronisation time (Woafo and Kreankel, 2002; Chembo and Woafo, 2002), define the synchronisation threshold parameters (Pyragas, 1998), and avoid loss of synchronisation, instability during the synchronisation, process (Osipov et al., 1997). On the other hand, optimisation is a key-word for wide-spread applications, and efforts should be made to minimise the synchronisation time. This problem is important in all fields where synchronisation finds or will find practical interests. Then, it is fair to say that there is a need to study adaptive chaos synchronisation and optimisation problems of nonlinear systems with uncertainties.

Two current questions are still open from the chaos synchronisation and suppression problem. The former is about how to set an arbitrary time in which the stabilisation of system be successfully achieved. The latter concerns to the estimation (and possibly reduction) of the control effort wasted during the execution of controller. On this issue, a research direction is being consolidated. Firstly, Femat et al. $(1999,2002)$ proposed an approach with low-parametrisation structure, which is a robust asymptotic feedback coupling (Femat et al., 2000). After that, Bowong et al. (2006) reported a scheme for the computation of the duration time departing from the robust asymptotic feedback coupling. However the control effort is not accounted.

In the light of recent developments in chaos synchronisation, this paper addresses the optimisation problem of the synchronisation of chaotic systems by accounting the control effort departing from advances reported in Femat et al. (1999, 2000, 2002, 2009) and Bowong et al. (2006). The main idea behind our proposal is, departing from the discrepancy synchronisation error system, to construct an extended nonlinear system which should be dynamically equivalent to the original system. In this way, the discrepancy is lumped into a nonlinear function, which is rewritten into the extended nonlinear system as a state variable. We then propose a robust adaptive feedback which accomplish the synchronisation of chaotic systems using an optimal tuning scheme based on Riccatti equations. A high-order sliding mode observer is used to get the estimates of the output and its time derivatives, and then the estimated values are used to design a robust adaptive feedback to force the dynamics of the slave system to follow the master system in spite of uncertainties errors, mismatch parameter and external perturbations. The proposed approach is algebraic and only requires to solve a matrix equation. 
Illustrative examples have been given to verify and validate analytical results. The results show that the control effort can be accounted under some conditions to set the time of convergence.

The rest of the paper is organised as follows. In the next section, a design of robust synchronisation scheme is proposed. In Section 3, some illustrative examples are presented to testify the performance of our proposed design. Finally, some conclusions are drawn in Section 4.

\section{Main result}

\subsection{Problem statement}

In this section, we describe the class of uncertain systems under study and state the synchronisation problem. The synchronisation objective is to design a robust direct feedback controller such that the slave system follows the master system at a finite time.

Let us consider the following nonlinear dynamical system whose dynamical behaviour is chaotic:

$$
\left\{\begin{array}{l}
\dot{x}_{m}=f\left(x_{m}, p\right) \\
y_{m}=h\left(x_{m}\right)
\end{array}\right.
$$

where $x_{m}=\left[x_{1 m}, x_{2 m}, \ldots, x_{n m}\right]^{\top} \in \mathbb{R}^{n}$ denotes the state vector of the master system, $f$ is an unknown smooth nonlinear function uniformly bounded in $t, p$ is the parameters vector, $y_{m} \in \mathbb{R}$ is the system output (measurable state) and $h$ is a smooth function.

Let us now take a chaotic dynamical system of the same order as equation (1):

$$
\left\{\begin{array}{l}
\dot{x}_{s}=f\left(x_{s}, q\right)+g\left(x_{s}\right) u, \\
y_{s}=h\left(x_{s}\right),
\end{array}\right.
$$

where $x_{s}=\left[x_{1 s}, x_{2 s}, \ldots, x_{n s}\right]^{\top} \in \mathbb{R}^{n}$ denotes the state vector of the slave system, $y_{s} \in \mathbb{R}$ the system's output, $f$ and $g$ are a nonlinear function with appropriate dimension, $q \neq p$ is the parameters vector and $u \in \mathbb{R}$ is the feedback coupling. As we deal with chaotic systems, equations (1) and (2) can be considered to be bounded.

System (1) is the so-called master, whereas system (2) represents the slave system. System (1) describes the goal dynamics while system (2) represents the experimental system to be controlled. The synchronisation problem can be stated as follows: given the transmitted signal $y_{m}$ and least prior information about the structure of the nonlinear filter, system (1), to design a feedback coupling $u$ which synchronises the orbits of both the master and slave systems at an established finite time, i.e.,

$$
\lim _{t \rightarrow T} x_{s}(t) \approx x_{m}(t)
$$

where $T$ is the synchronisation established time.

From the control viewpoint, the synchronisation problem can be seen as follows. Let us define the synchronisation error as follows 


$$
e_{i}=x_{s}-x_{m}
$$

Then, the dynamics of the synchronisation error is given by

$$
\left\{\begin{array}{l}
\dot{e}=\Delta f\left(x_{m}, x_{s}, p, q\right)+g\left(e, x_{m}\right) u \\
y=h\left(e, x_{m}\right)
\end{array}\right.
$$

where $\Delta f=f\left(x_{s}, q\right)-f\left(x_{m}, p\right)$ is a smooth vector field, and $h\left(e, x_{m}\right)=h\left(e+x_{m}\right)-h\left(x_{m}\right)$ is a smooth function which determines the difference between the master and slave outputs. In this way, the synchronisation problem can be seen as the stabilisation of equation (5) at the origin in a finite horizon. In other words, the problem is to find a robust feedback coupling law $u(t)$ such that $\lim _{t \rightarrow T}\|e(t)\| \approx 0$ (which implies that $x_{s} \approx x_{m}$ for all $t \geq T>0$ ).

First, since the functions $f\left(x_{s}, q\right), f\left(x_{m}, p\right)$ and $h$ are sufficiently smooth, and the output $y$ is assumed to have a relative degree $\rho$ for all $(e, u) \in \Omega \times \mathbb{R}$ where $\Omega \subset \mathbb{R}^{n}$, there exists an invertible change of coordinates $z=\Phi(e)$ such that the synchronisation error system (5) can be written in the following canonical form (Isidori, 1989; Kocarev et al., 1998; Femat et al., 1999; Femat, 2002):

$$
\left\{\begin{array}{l}
\dot{z}_{i}=z_{i+1}, \quad i=1,2, \ldots, \rho-1 \\
\dot{z}_{\rho}=\alpha(z, \zeta)+\beta(z, \zeta) u, \\
\dot{\zeta}=\Psi(z, \zeta), \\
y=z_{1},
\end{array}\right.
$$

where $y$ is the system output, $\rho$ the relative degree of system (6) ( $\rho$ is equal to the lowest order time-derivative of the output $y$ that is directly related to the feedback coupling $u$ ) and $\zeta \in \mathbb{R}^{n-\rho}$ is the unobservable states vector (internal dynamics).

To ensure the achievement of the synchronisation's objective, we make the following assumptions.

1 There is only a single system state as the output. Without loss of generality, it is letting $y_{m}=h\left(x_{m}\right)=x_{1 m}$, i.e., only the first system state is measurable.

2 There are some unknown model mismatches between the master and the slave, i.e., $\Delta f=f\left(x_{s}, q\right)-f\left(x_{m}, p\right) \neq 0$.

3 The error dynamics system can be transformed into a canonical form, i.e., there exists a diffeomorphism transformation of coordinate given by system (5).

4 System (5) is minimum phase system.

Some comments regarding the above assumptions are in order. The first assumption is realistic. For instance, in the secure communication case, only the transmitted signal and receiver signal are available for feedback from measurements. Another example can be found in neuron synchronisation where master neuron transmits a scalar signal. The slave neuron tracks the signal of the master neuron. Concerning the second assumption, we claim that it is a general and practical situation because the mismatches between the master and the slave are unavoidably existed in real cases, and hence $\Delta f$ always exists. The source of such uncertainties are: 
1 unknown values of the model parameters or time-varying parameters

2 since the master and slave systems are chaotic, their trajectories depend on the initial conditions which are often unknown

3 structural differences between models of the master and slave systems.

Concerning the third assumption, several systems subjected to chaotic synchronisation can be transformed into the canonical form (6). For example, the Lorenz dynamical and several types of Chua's circuits can be transformed into the canonical form (6) with a relative degree $\rho<n$. Also non-autonomous second-order chaotic systems such as Duffing and Van der Pol oscillators can be written as the canonical form (6) with $\rho=n$. Regarding the last assumption, the minimum phase assumption implies that the zero dynamics $\dot{\zeta}=\Psi(0, \zeta)$ converges to the origin. In other words, the closed-loop system is internally stable (Kocarev et al., 1998). From the control viewpoint this is a strong assumption. But this is reasonable for the boundedness of the chaotic attractor in the state space and the interaction of all the trajectories inside the attractor in region Y. So when we have taken actions to achieve $\lim _{t \rightarrow T} z_{i}(t)=0$, the part $\Psi(z, \zeta) \rightarrow \Psi(0, \zeta) \rightarrow 0$ as $t \rightarrow \infty$ asymptotically for the so-called minimum-phase character. However, several interesting chaotic systems satisfy this internal stability assumption.

Now, since $f\left(x_{s}, q\right)$ and $f\left(x_{m}, p\right)$ are uncertain, the mapping $z=\Phi(e)$ is an uncertain nonlinear change of coordinates, hence $\alpha(z, \zeta)$ and $\beta(z, \zeta)$ in the transformed system (6) are also unknown. The idea to deal with the uncertain terms $\alpha(z, \zeta)$ and $\beta(z, \zeta)$ is to lump them into a new function which can be interpreted as a new observable state. By an observable state we mean that the dynamics of such state can be reconstructed from online measurements (for example $y=z_{1}$ ). Thus, let us suppose that $\operatorname{sgn}\left[\beta_{E}(z)\right]$ is equal to $\operatorname{sgn}[\beta(z, \zeta)]$ where $\operatorname{sgn}()$ stands for the sign function. In addition, let $\eta=z_{\rho+1}=\Theta(z, \zeta, u)$ with $\Theta(z, \zeta, u)=\alpha(z, \zeta)+\delta(z, \zeta) u$ and $\delta(z, \zeta)=\beta(z, \zeta)-\beta_{E}(z)$. In this way, system (5) can be rewritten in the following extended form (Femat et al., 1999, 2009; Femat, 2002; Femat and Solís-Perales, 2008):

$$
\left\{\begin{array}{l}
\dot{z}_{i}=z_{i+1}, \quad i=1,2, \ldots, \rho-1, \\
\dot{z}_{\rho}=\eta+\beta_{E}(z) u, \\
\dot{\eta}=\Gamma(z, \eta, \zeta, u, \dot{u}), \\
\dot{\zeta}=\Psi(z, \zeta), \\
y=z_{1},
\end{array}\right.
$$

where

$$
\begin{aligned}
\Gamma(z, \eta, \zeta, u, \dot{u})=\sum_{i=1}^{\rho-1} & {\left[z_{i+1} \partial_{i} \Theta(z, \zeta, u)\right]+\left[\eta+\beta_{E}(z)\right] \partial_{\rho} \Theta(z, \zeta, u) } \\
& +\delta(z, \zeta) \dot{u}+\partial_{\zeta} \Theta(z, \zeta, u) \Psi(z, \zeta)
\end{aligned}
$$


with $\partial_{i} \Theta(z, \zeta, u)=\partial \Theta(z, \zeta, u) / \partial z_{i}, i=1, \ldots, \rho$. From system (7), it is to see that the mismatches between the master and slave have been lumped into a nonlinear function $\eta=\Theta(z, \zeta, u)$, which has been interrupted as an augmented state variable $z_{\rho+1}$.

The scalar function $u$ and a state estimator for the states $(z, \eta)$ can be designed from the system (7). This fact can be proved because the system (7) is dynamically externally equivalent to system (6) (Femat et al., 1999, 2009; Femat, 2002; Femat and Solís-Perales, 2008).

\subsection{Optimal robust control design}

To achieve the finite time synchronisation stated in the previous section, a suitable robust feedback $u$ will be designed. In what follows, the problem of designing $u$ is addressed in such a manner that energy wasted by the feedback is accounted. Towards the optimisation, the first step in our approach is to consider the transitive of states. To this end, the following quadratic criterion is defined by quantifying the transient trajectory of the synchronisation error (Anderson and Moor, 1990):

$$
J(z, u)=z^{T}(T) Q_{f} z(T)+\int_{t_{0}}^{T} z^{T}(t) Q z(t) d t,
$$

where $t_{0} \geq 0$ is the time at which the control starts and $T>t_{0}$ is the time for which the synchronisation error system (7) achieves the desired trajectory ( $e=0$ ); $Q>0$ and $Q_{f} \geq 0$ are positive symmetric matrices. This paper deals with the problem of finding a control law $u$ which synchronises both the master (1) and slave (2) systems at an established finite time $T$ meanwhile minimising the cost function (8).

The feedback coupling is designed as follows:

$$
u(z)=\frac{-1}{\beta_{E}(z)}\left[\eta+\frac{1}{2} B^{\top} P z\right], \quad t_{0} \leq t \leq T,
$$

where $T$ is given, $P \in \mathbb{R}^{\rho \times \rho}$ is a symmetric positive matrix, solution of the Riccati equation:

$$
\left\{\begin{array}{l}
-\dot{P}=A^{\top} P+P A-P B B^{\top} P+Q, \\
P(T)=Q_{f},
\end{array}\right.
$$

with $A \in \mathbb{R}^{\rho \times \rho}$ and $B \in \mathbb{R}^{\rho}$ two matrices defined as

$$
A=\left[\begin{array}{cccccc}
0 & 1 & 0 & 0 & \ldots & 0 \\
0 & 0 & 1 & 0 & \ldots & 0 \\
\vdots & \vdots & \ddots & \ldots & \ddots & \vdots \\
0 & 0 & 0 & 0 & \ldots & 1 \\
0 & 0 & 0 & 0 & \ldots & 0
\end{array}\right] \text { and } B=\left[\begin{array}{c}
0 \\
\vdots \\
0 \\
1
\end{array}\right]
$$


From optimal control theory (Anderson and Moor, 1990), it is known that the linear optimal control depends mainly on the relative sizes of the system state and the control weighting matrices $Q$ of the quadratic functional. We also refer the reader in Femat et al. (2009).

We have the following result.

Proposition 1. The state feedback control (9) stabilises the error system (7) in the sense that the solution $(z(t), \eta(t), \zeta(t)) \rightarrow\left(0, \eta^{*}, \zeta^{*}\right)$, where $\zeta^{*}$ and $\eta^{*}=\alpha\left(0, \zeta^{*}\right)+\delta\left(0, \zeta^{*}\right) u^{*}$ are respectively vector and scalar constant, as $t \rightarrow T<\infty$, for any initial conditions $\left(\mathrm{z}_{0}, \eta_{0}, \zeta\right) \in U^{0} \times \Xi \subset \mathbb{R}^{\mathrm{n}} \times \mathbb{R}$, where $\Xi=\left\{\eta_{0} \in \mathbb{R}: \eta_{0}=\Theta\left(\mathrm{z}_{0}, \zeta_{0}, u^{*}\right)\right\}$, with suitable matrices $Q$ and $Q_{f}$. Moreover, the closed loop performs a value of the functional (8) $J(z, u)=z_{0}^{\top} P(0) z_{0}$, where $z_{0}$ is the initial condition of the error system (7).

Proof. Substituting the feedback control law (9) in the error system (7), the closed-loop can be written as

$$
\left\{\begin{array}{l}
\dot{z}=\left(A-\frac{1}{2} B B^{\top} P\right) z, \\
\dot{\eta}=\Gamma(z, \eta, \zeta, u, \dot{u}),
\end{array}\right.
$$

and

$$
\dot{\zeta}=\Psi(z, \zeta)
$$

By the Assumption 2, the subsystem (12) is stable as $(z, \eta) \rightarrow(0,0)$ for any $(z(0), \eta(0)$, $\zeta(0)$ ) in a subset $U^{0} \in \mathbb{R}^{\mathrm{n}}$ containing the (regular) point $e^{0} \in \mathbb{R}^{\mathrm{n}}$. Therefore, the proof is focused on the first equation of (11). The time dependence has been omitted in this proof for seeking simplicity in notation.

Consider the following Lyapunov candidate function:

$$
V=z^{\top} P z
$$

Its time derivative satisfies

$$
\begin{aligned}
\dot{V} & =\dot{z}^{\top} P z+z^{\top} \dot{P}(t) z+z^{\top} P \dot{z}, \\
& =z^{\top}\left(A-\frac{1}{2} B B^{\top} P\right)^{\top} P z+z^{\top} P\left(A-\frac{1}{2} B B^{\top} P\right) z \\
& =z^{\top}\left(-A^{\top} P-P A+P B B^{\top} P-Q\right) z, \\
& =-z(t)^{\top} Q z(t) .
\end{aligned}
$$

which is negative definite. Hence, the state $z(t)$ converges to zero for all $t$. This implies that $z=0$ is a stable point. Since $z(t)$ and $\zeta(t)$ belong to some attractor, functions $\alpha(z, \zeta)$ and $\beta(z, \zeta)$ in the error system (7) are smooth and bounded, hence, the function $\Gamma(z, \eta, \zeta, u, \dot{u})$ is also bounded and smooth. Moreover, the state $\zeta(t)$ under the state feedback is bounded and converges to zero since the system is in cascade form. In addition, the matrix $Q$ determines the convergence rate. Then, by integrating equation (14) from $t_{0}$ to $T$ and by using equation (13), we have 


$$
z(T)^{\top} P z(T)-z(0)^{\top} P(0) z(0)=-\int_{t_{0}}^{T} z(t)^{\top} Q z(t) d t
$$

from where $J(z, u)=z_{0}^{\top} P(0) z_{0}$. This completes the proof.

We stress that the feedback (9) is defined in the interval $t_{0} \leq t \leq T$ and the stabilisation is achieved for some matrices $Q$ and $Q_{f}$ if solutions of equation (10) exist in such interval. Also, note that the proposed feedback requires availability of the complete state. This can be seen as drawback; hence an approach of the state feedback is required to avoid dependence on the full information of the system. Additionally, other approach to derive result in Proposition 1 can be stated by introducing the following definitions $\tilde{B}=\left[0 \ldots 0, \beta_{E}(z)\right]^{\top}$ with $u_{u}=-\frac{\eta}{\beta_{E}(z)}$. The control $u_{u}$ can be named the 'unavoidable' part of feedback control because it represents the force necessary to compensate the non-linear function $\eta$. In this sense, as a second step is derived to design the feedback such that the control effort can be accounted. That is, the criterion (8) can be re-defined to include the 'avoidable' control effort as follows:

$$
\tilde{J}(z, \tilde{u})=z(t)^{\top} Q_{f} z(t)+\int_{t_{0}}^{T}\left[z(t)^{\top} Q z(t)+\tilde{u}^{\top} R \tilde{u}\right] d t,
$$

with a given symmetric matrix $R>0$ and $\tilde{u}=u-u_{u}$. Hence, the control law becomes

$$
u_{u}=-\frac{\eta}{\beta_{E}(z)}+\tilde{u}
$$

Therefore, the closed loop takes the form:

$$
\left\{\begin{array}{l}
\dot{z}=A z+\tilde{B} \tilde{u}, \\
\dot{\eta}=\Gamma(z, \eta, \zeta, u, \dot{u}), \\
\dot{\zeta}=\Psi(z, \zeta) .
\end{array}\right.
$$

which allows to set the standard LQ problem as in Anderson and Moor (1990):

$$
\min _{u} \tilde{J}(z, \tilde{u}),
$$

such that

$$
\dot{z}=A z+\tilde{B} \tilde{u},
$$

whose solution for controller is given by

$$
\tilde{u}=-R^{-1} B^{\top} P(t) z, \quad t_{0} \leq t \leq T,
$$

where $P(t)$ is now the solution of the following Riccati equation:

$$
\left\{\begin{array}{l}
-\dot{P}=A^{\top} P+P A-P \tilde{B} R^{-1} \tilde{B}^{\top}+Q, \\
P(T)=Q_{f},
\end{array}\right.
$$


and a value of functional (16) given by

$$
\tilde{J}(z, \tilde{u})=z_{0}^{\top} P(0) z_{0} .
$$

We stress that, at this point, the full knowledge of states is required by this approach. Nevertheless, the linearising feedback control law (9) is not physically realisable because it requires measurements of the state $z$ and the uncertain state $\eta$ which is a very stringent demand for the literature of chaotic secure communication. In order to increase the security of communication, the least possible information about the transmitter should be contained in the communication channel. According to the Assumption 1, only the state $z_{1}$ is available for feedback. Some strategies have been developed to achieve this, such as the linearising feedback-based estimator (Femat et al., 1999, 2009; Femat and Solís-Perales, 2008), the nonlinear geometric-based estimator (Li et al., 2005), and so on. In the next section, the full knowledge situation is relaxed by using a state estimator. The cost to pay is a higher control effort because of estimation than those due to the use of all states in feeding back.

\subsection{A suboptimal robust feedback coupling}

There are two basic reasons to relax the optimal condition presented previously. The former, recall that the optimal result in the previous section concerns to the perfect knowledge of the whole state, the dynamics and the augmented state $\eta$, therefore if we consider the Assumption 1, the optimality of the previous section is not achieved perfectly. The latter concerns to the controversy on the optimal, the robust and the fragility notion in feedback interconnections (Keel and Bhattacharyya, 1997, 1998; Mäkilä, 1998; Femat, 2002). Then, the construction of an state estimator yields in suboptimal robust approach. As it has been established in Femat et al. (1999, 2009), Femat (2002) and Femat and Solís-Perales (2008), the problem of estimating $(z, \eta)$ can be addressed by using a high-gain observer. Here, the dynamics of the states $z$ and $\eta$ can be reconstructed from measurements of the output $y=z_{1}$ by using the following high-order sliding mode-based time differentiator estimating systems:

$$
\left\{\begin{array}{l}
\dot{\hat{z}}_{1}=\hat{z}_{2}-L \lambda_{1}\left|\hat{z}_{1}-z_{1}\right|^{\frac{\rho}{\rho+1}} \operatorname{sgn}\left(\hat{z}_{1}-z_{1}\right), \\
\dot{\hat{z}}_{i}=\hat{z}_{i+1}-L^{i} \lambda_{i}\left|\hat{z}_{1}-z_{1}\right|^{\frac{\rho-i+1}{\rho-i+2}} \operatorname{sgn}\left(\hat{z}_{1}-z_{1}\right), \quad 1<i<\rho, \\
\dot{\hat{z}}_{\rho}=\hat{\eta}-L^{\rho} \lambda_{\rho}\left|\hat{z}_{1}-z_{1}\right|^{\frac{1}{2}} \operatorname{sgn}\left(\hat{z}_{1}-z_{1}\right)+\beta_{E}(\hat{z}) u, \\
\dot{\hat{\eta}}=-L^{\rho+1} \lambda_{\rho+1} \operatorname{sgn}\left(\hat{z}_{1}-z_{1}\right),
\end{array}\right.
$$

where $L>0$ is the so-called high-gain parameter, $\lambda_{i}, i=1, \ldots, \rho+1$ and $\beta_{E}(\hat{z})$ are suitable selected positive constants.

Let $\tilde{e}_{i} \in \mathbb{R}^{\rho+1}$ be the estimation error vector whose components are defined as follows: $\tilde{e}_{i}=\hat{z}_{i}-z_{i}, i=1, \ldots, \rho$ and $\tilde{e}_{\rho+1}=\hat{\eta}-\eta$. By subtracting $(\rho+1)^{\text {th }}$ subsystem of system (7) and system (24), one has 


$$
\left\{\begin{array}{l}
\dot{\tilde{e}}_{1}=\tilde{e}_{2}-L \lambda_{1}\left|\tilde{e}_{1}\right|^{\frac{\rho}{\rho+1}} \operatorname{sgn}\left(\tilde{e}_{1}\right), \\
\dot{\tilde{e}}_{i}=\tilde{e}_{i+1}-L^{i} \lambda_{i}\left|\tilde{e}_{1}\right|^{\frac{\rho-i+1}{\rho-i+2}} \operatorname{sgn}\left(\tilde{e}_{1}\right), \quad 2 \leq i \leq \rho, \\
\dot{\tilde{e}}_{\rho}=\tilde{e}_{\rho+1}-L^{\rho} \lambda_{\rho}\left|\tilde{e}_{1}\right|^{\frac{1}{2}} \operatorname{sgn}\left(\tilde{e}_{1}\right), \\
\dot{\tilde{e}}_{\rho+1}=-L^{\rho+1} \lambda_{\rho+1} \operatorname{sgn}\left(\tilde{e}_{1}\right)-\Gamma(.),
\end{array}\right.
$$

We define the following variables

$$
\left\{\begin{array}{l}
w_{1}=L^{\rho}\left|\tilde{e}_{1}\right|^{\frac{\rho}{\rho+1}} \operatorname{sgn}\left(\tilde{e}_{1}\right), \\
w_{i}=L^{\rho+1-i}\left|\tilde{e}_{1}\right|^{\frac{\rho}{\rho+1}-\frac{\rho-i+1}{\rho-i+2}} \tilde{e}_{i}, \quad 2 \leq i \leq \rho, \\
w_{\rho+1}=\tilde{e}_{\rho+1} .
\end{array}\right.
$$

Note that

$$
\dot{w}_{1}=L^{\rho}\left[\left|\tilde{e}_{1}\right|^{\frac{\rho}{\rho+1}} \operatorname{sgn}\left(\tilde{e}_{1}\right)\right]\left(\dot{\hat{z}}_{1}-\dot{z}_{1}\right),
$$

where superscript [.] denotes the first derivative of time. Due to the boundedness of chaotic systems, $z_{1}$ is bounded. Therefore, to track the dynamics of $z_{1}, \hat{z}_{1}$ must be bounded too. Then, it can be derived that $\left[\left|\tilde{e}_{1}\right|^{\frac{\rho-i+1}{\rho-i+2}} \operatorname{sgn}\left(\tilde{e}_{1}\right)\right], i=1, \ldots, \rho$ is also bounded. Without loss of generality, assuming that its upper bound is $\varepsilon$, which can be chosen as a large enough number, then we get $\dot{w}_{1} \leq L^{\rho} \varepsilon\left(\dot{\hat{z}}_{1}-\dot{z}_{1}\right)$. We point out that the value of $\varepsilon$ is unknown in practice. Generally, it can be approximated based on the prior information of the system upper bound. In this way, system (26) can be changed into the following 'estimation system':

$$
\dot{\bar{w}}=L D \bar{w}+\Omega(.)
$$

where $\bar{w}=\left(w_{1}, \ldots, w_{\rho+1}\right)^{T}$ and $\Omega()=.[0,0, \ldots,-\Gamma(.)]^{T}$ and $D$ is defined as follows

$$
D=\left[\begin{array}{ccccc}
-\lambda_{1} \varepsilon & \varepsilon & 0 & \ldots & 0 \\
-\lambda_{2} & 0 & 1 & \ldots & 0 \\
\vdots & \vdots & \vdots & \ddots & \vdots \\
-\lambda_{\rho} & 0 & 0 & \ldots & 1 \\
-\lambda_{\rho+1} & 0 & 0 & \ldots & 0
\end{array}\right]
$$


Since system (7) is contained in an attractor, the time derivative of $\dot{z}_{\rho}$ is bounded and thus $\Gamma$ (.) is also a bounded function. Thus, by choosing suitable constants $\lambda_{i}, i=1, \ldots$, $\rho+1$ such that the matrix $D$ has all its eigenvalues in the left-half complex plane, we have $w_{i} \rightarrow 0,1 \leq i \leq \rho+1$. That is, the 'estimating error' system $\bar{w}$ is globally asymptotically stable at zero, which implies that $\hat{z}_{1} \rightarrow z_{i}, 1<i \leq \rho+1$. So, the estimates of unmeasurable state $z_{i}, 1<i \leq \rho$ and model uncertainties $\eta$ can be duly obtained.

By using the estimated values $(\hat{z}, \hat{\eta})$, the feedback (9) can be written as

$$
u(\hat{z})=\frac{-1}{\beta_{E}(\hat{z})}\left[\hat{\eta}+\frac{1}{2} B^{\top} P \hat{z}\right], \quad t_{0} \leq t \leq T .
$$

while, after transient, the feedback can be expressed by

$$
u(\hat{z})=\frac{-1}{\beta_{E}(\hat{z})}\left[\hat{\eta}+\frac{1}{2} B^{\top} \bar{P} \hat{z}\right], \quad t>T,
$$

where $\bar{P}$ is a constant positive definite matrix such that the error system (7) remains in $e$ $=0$ for $t>T$, thus $\bar{P}$ is also a tuning parameter. Notice that the control effort on the suboptimal robust controller is higher than the ideal one. As a matter of fact, the waste of energy increases due to the estimation since

$$
\tilde{J}(z, \tilde{u})=z_{0}^{\top} P(0) z_{0}+\int_{t_{0}}^{T}(z-\hat{z})^{\top} Q(z-\hat{z}) .
$$

as a consequence, the choice of parameters becomes important and present a trade-off between optimisation and estimation. The tuning parameter $\bar{P}$ is also very important because it can be adjusted after the achievement of the synchronisation $(t>T)$. Therefore, it permits to reduce and keep the error system to zero in case of loss of synchronisation.

\section{Illustrative examples}

In this section, the implementation of the synchronisation of two chaotic systems via the proposed robust adaptive feedback combined with the high-order sliding mode observer is illustrated. The underlying idea is to evaluate the performance of the proposed robust synchronisation strategy. In the numerical simulations, the program uses the Runge-Kutta integration algorithm which is one classical method for ordinary differential equations.

\subsection{Synchronisation of two Chua's oscillators}

Here, we study the synchronisation of two Chua's oscillators with parameter mismatching. The aim is to show that the synchronisation can be attained in spite of parametric variation and to illustrate that the chaotic minimum-phase assumption is satisfied. The Chua oscillator is an electronic circuit which consists of one linear inductor, two linear resistors, two linear capacitors and a nonlinear resistor which is the so-called Chua diode. It is a typical chaotic oscillator with its dynamics governed by the following equation: 


$$
\left\{\begin{array}{l}
\dot{x}_{1 m}=p_{1}\left(x_{2 m}-x_{1 m}-f\left(x_{1 m}\right)\right), \\
\dot{x}_{2 m}=x_{1 m}-x_{2 m}+x_{3 m}, \\
\dot{x}_{3 m}=-p_{2} x_{2 m}
\end{array}\right.
$$

where

$$
f\left(x_{1 m}\right)=b x_{1 m}+0.5(a-b)\left(\left|x_{1 m}+1\right|-\left|x_{1 m}-1\right|\right) .
$$

The slave system can be constructed as:

$$
\left\{\begin{array}{l}
\dot{x}_{1 s}=q_{1}\left(x_{2 s}-x_{1 s}-f\left(x_{1 s}\right)\right)+u, \\
\dot{x}_{2 s}=x_{1 s}-x_{2 s}+x_{3 s}, \\
\dot{x}_{3 s}=-q_{2} x_{2 s},
\end{array}\right.
$$

where

$$
f\left(x_{1 s}\right)=b x_{1 s}+0.5(a-b)\left(\left|x_{1 s}+1\right|-\left|x_{1 s}-1\right|\right) .
$$

Subtracting equation (31) from equation (32) and using the notation $e_{1}=x_{1 s}-x_{1 m}$, $e_{2}=x_{2 s}-x_{2 m}$ and $e_{3}=x_{3 s}-x_{3 m}$ yields

$$
\left\{\begin{array}{l}
\dot{e}_{1}=\Delta F+u, \\
\dot{e}_{2}=e_{1}-e_{2}+e_{3}, \\
\dot{e}_{3}=-q_{2} e_{2}+G,
\end{array}\right.
$$

where $\Delta F$ and $G$ are unknown functions defined as follows:

$$
\begin{aligned}
& \Delta F=q_{1}\left(e_{2}-e_{1}\right)+\left(q_{1}-p_{1}\right) x_{2 m}+\left(p_{1}-q_{1}\right) x_{1 m}+p_{1} f\left(x_{1 m}\right)-q_{1} f\left(e_{1}+x_{1 m}\right) \\
& \text { and } G=\left(p_{2}-q_{2}\right) x_{2 m} .
\end{aligned}
$$

Now, defining the master output by $y_{m}=x_{1 m}$ and the slave output by $y_{s}=x_{1 s}$, one has that $y=h(e)=e_{1}$. This implies that the smallest integer is $\rho=1$. In this way, the coordinates transformation is globally defined by $z_{1}=e_{1}, \zeta_{1}=e_{2}$ and $\zeta_{2}=e_{3}$. Then, the uncertain system (33) can be rewritten as follows:

$$
\left\{\begin{array}{l}
\dot{z}_{1}=\Delta F+u, \\
\dot{\zeta}_{1}=z_{1}-\dot{\zeta}_{1}+\dot{\zeta}_{2}, \\
\dot{\zeta}_{2}=-q_{2} \dot{\zeta}_{1}+G, \\
y=z_{1},
\end{array}\right.
$$

where $y$ denotes the output of the error uncertain system. In order to illustrate that system (34) satisfies the minimum phase assumption, one may prove that $\dot{\zeta}_{1}=z_{1}-\dot{\zeta}_{1}+\dot{\zeta}_{2}$ and $\dot{\zeta}_{2}=-q_{2} \dot{\zeta}_{1}+G$ converge asymptotically to zero when $z_{1}=0$. Note that $\zeta=\left(\zeta_{1}, \zeta_{2}\right)^{T}$ is uncertain. However, it is clear that $\zeta$ is bounded. Thus, the zero dynamics can be written as 


$$
\dot{\zeta}=E \zeta+H
$$

where $H=[0, G]^{T}$ and

$$
E=\left[\begin{array}{ll}
-1 & 1 \\
-q_{2} & 0
\end{array}\right]
$$

which is Hurwitz because $q_{2}>0$. Since $G$ is bounded, the zero dynamics subsystem $\dot{\zeta}=E \zeta+H$ is asymptotically stable. Hence, system (34) is minimum phase. Since Assumptions 1 and 2 are satisfied, the dynamical system (34) can be transformed into its equivalent canonical form (6) (with $\eta=\Delta F$ as the augmented state). So, the extended state observer (24) can be described in the following form:

$$
\left\{\begin{array}{l}
\dot{\hat{z}}_{1}=\hat{\eta}-L \lambda_{1}\left|\hat{z}_{1}-z_{1}\right|^{\frac{1}{2}} \operatorname{sgn}\left(\hat{z}_{1}-z_{1}\right)+u, \\
\dot{\hat{\eta}}=-L^{2} \lambda_{2} \operatorname{sgn}\left(\hat{z}_{1}-z_{1}\right) .
\end{array}\right.
$$

Hence, the robust adaptive feedback law (28) and (29) can be described as

$$
u(\hat{z})= \begin{cases}0 & t<t_{0}, \\ -\hat{\eta}-\frac{1}{2} B^{\top} P \hat{z}_{1} & t_{0} \leq t \leq T, \\ -\hat{\eta}-\frac{1}{2} B^{\top} \bar{P} \hat{z}_{1} & t>T\end{cases}
$$

In the numerical simulations, the initial conditions of the master and slave systems were chosen to be $\left(x_{1 m}(0), x_{2 m}(0), x_{3 m}(0)\right)=(0.1,0.02,0.001)$ and $\left(x_{1 s}(0), x_{2 s}(0), x_{3 s}(0)\right)$ $=(0.2,1,2)$. The initial condition of $(\hat{z}, \hat{\eta})$, is chosen to be $\left(\hat{z}_{1}(0), \hat{\eta}(0)\right)=(0.1,0)$.

The true parameter values of the master are: $p_{1}=9$ and $p_{2}=100 / 7$, while the parameter values of the slave are: $q_{1}=8.82, q_{2}=14$ corresponding to $2 \%$ of parameter mismatches. The parameter values of piecewise-linear nonlinear resistor are $a=-8 / 7$ and $b=-5 / 7$. The high gain parameter value was chosen as $L=20$ and the constants are chosen as: $\lambda_{1}=1, \lambda_{2}=2$. The controller parameters were $Q=40,000, Q_{f}=200$ and $\bar{P}=200$. The finite horizon is established at $T=0.1 \mathrm{sec}$ (i.e., the convergence should be attained at time $t \equiv T$ ).

Figure 1 provides a graphical representation of the synchronisation process between two Chua oscillators obtained numerically. From this figure, one can observe that the synchronisation error is stabilised at the origin by the output-feedback controller (35) and (36) in spite of the fact that both master and slave circuits have different parameters values. From Figure 1(b), one can see that a fairly good convergence of $e_{1}$ is obtained in about $0.1 \mathrm{sec}$ which corresponds to the finite horizon. Note that although the control input is acting only on the state $e_{1}$, the synchronisation errors $e_{2}$ and $e_{3}$ are also stabilised at the origin. 
Figure 1 Numerical simulation results of synchronisation between two Chua oscillators, (a) chaotic attractor corresponding to the master system during synchronisation; dynamics of the synchronisation errors with $2 \%$ of parameter mismatches (b) $e_{1}=x_{1 s}-x_{1 m}$ (c) $e_{2}=x_{2 s}-x_{2 m}$ (d) $e_{3}=x_{3 s}-x_{2 m}$

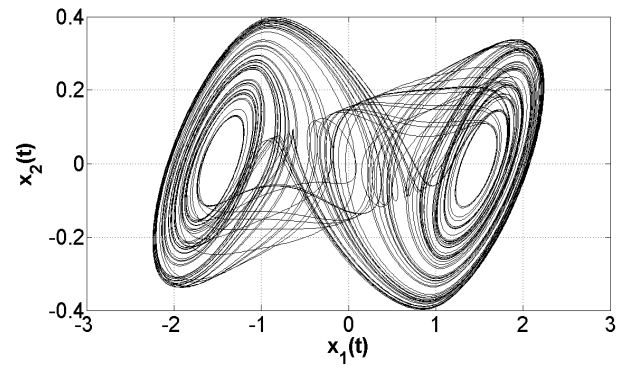

(a)

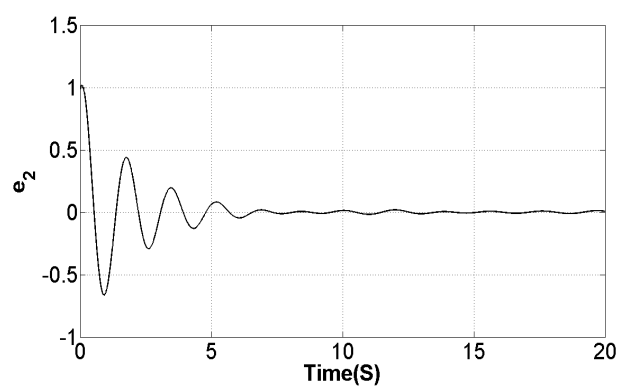

(c)

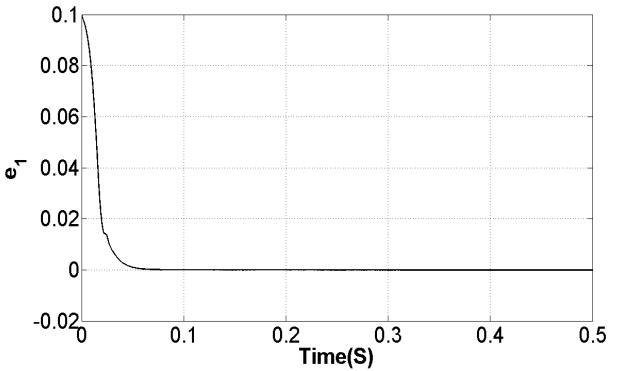

(b)

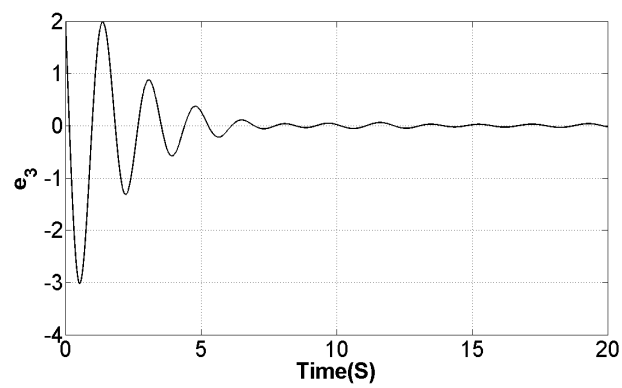

(d)

The time evolution of the control signal is depicted in Figure 2(a). From this figure, it is evident that the control signal is also stabilised at the origin. In order to add evidence of the effectiveness and efficiency of the proposed robust adaptive feedback, we have plotted in Figures 2(b) to 2(d) the phase portrait of the state variables of slave versus the state variables of master. It is evident that the manifolds $x_{1 s}=x_{1 m}, x_{2 s}=x_{2 m}$ and $x_{3 s}=x_{3 m}$ are stable, and one can conclude that the chaotic oscillations of the drive and response systems are synchronised in the complete sense and our synchronisation objective has been attained.

Now let us investigate the robustness of the proposed scheme in front of noise and mismatches. we suppose that the signal coming from the master $x_{1 m}$ is intrinsically modified by the channel noise and thus the channel output signal form is $\operatorname{awgn}\left(x_{1 m}, \operatorname{snr}\right)$, where snr represent the signal-noise rate. Figure 3 shows the behaviour of the errors norm $\left|e_{1}\right|$ with numerical time and the signal-noise ratio $s n r$ with $2 \%$ of parameter mismatch between the master and slave systems. It appears from this $3 D$ graph that, when snr increases the synchronisation is better. One can also observe that, a fairly good convergence of $\left|e_{1}\right|$ can be obtained in spite of the simultaneous presence of the noise and parameter mismatch between the master and slave systems. 
Figure 2 Performance of the proposed feedback control $u(t)$, (a) phase portraits of (b) $x_{1 s}$ versus $x_{1 m}$, (c) $x_{2 s}$ versus $x_{2 m}$ and (d) $x_{3 s}$ versus $x_{3 m}$

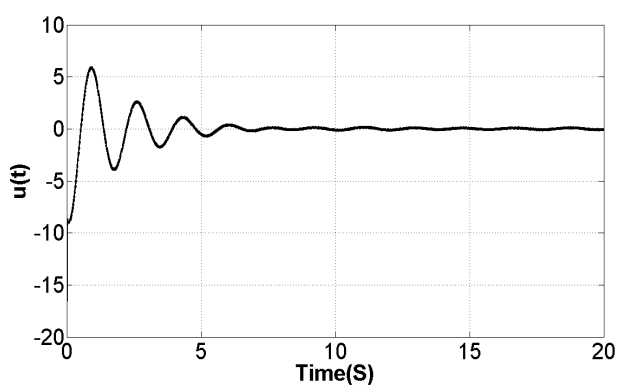

(a)

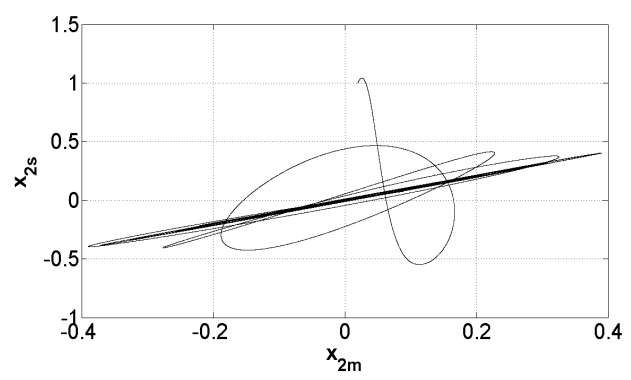

(c)

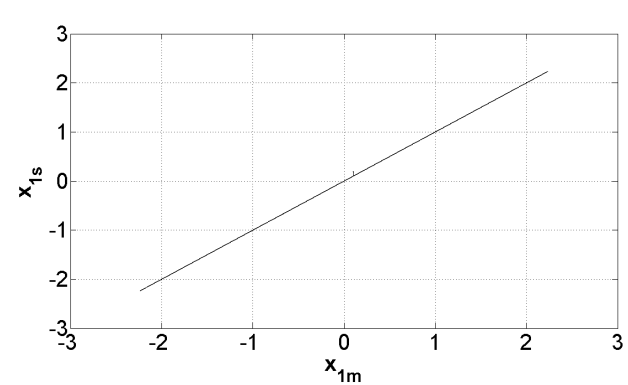

(b)

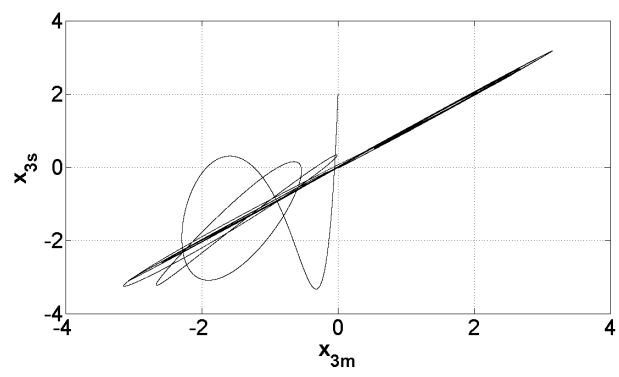

(d)

Figure 3 Time evolution of error norm $\left|e_{1}\right|$ when $s n r$ is varied (see online version for colours)

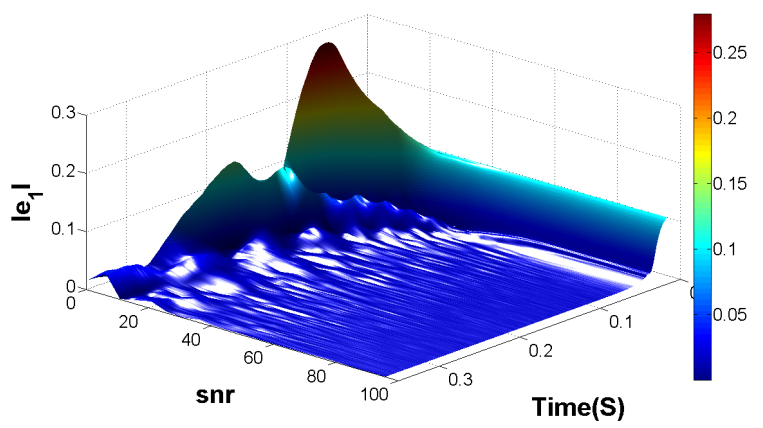

In comparison, one more simulation study is conducted to show the effect of the controller parameters on the time evolution of synchronisation error norm $\left|e_{1}\right|$ (because the controller is applied only on this state variable). Then without loss of generality, we set $Q_{f}=\bar{P}=\sqrt{Q}$ to appreciate the simultaneous influence of all the controller parameters when only $Q$ is varied. Figure 4 shows the effect of the controller parameters on the time evolution of error norm $\left|e_{1}\right|$ when $Q$ is varied. As expected, the error norm $\left|e_{1}\right|$ and the synchronisation time $T$ decreases as parameter $Q$ increases and vice versa. Therefore one can conclude that, when the parameter $Q$ increases the synchronisation is better. 
Figure 4 Time evolution of error norm $\left|e_{1}\right|$ when parameter $Q$ is varied (see online version for colours)

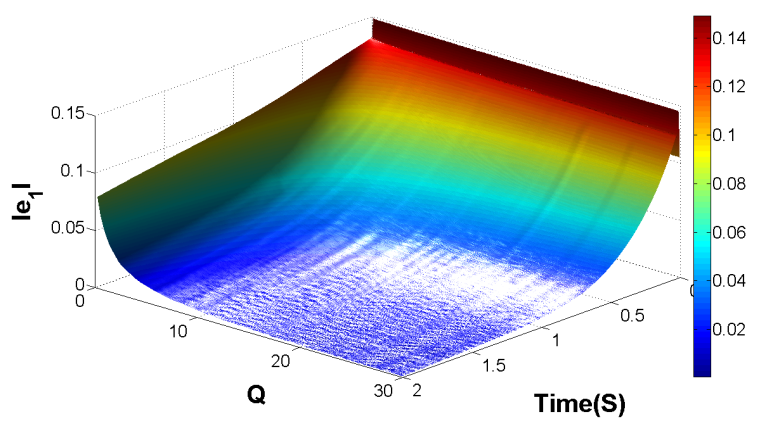

\subsection{Synchronisation of two Lorenz oscillators}

Herein, we take the Lorenz system as our second illustrative example, which has been frequently studied for synchronisation of chaotic systems. The Lorenz system is a typical chaotic oscillator and its dynamics governed by the following equation:

$$
\left\{\begin{array}{l}
\dot{x}_{1 m}=p_{1}\left(x_{2 m}-x_{1 m}\right) \\
\dot{x}_{2 m}=p_{2} x_{1 m}-x_{2 m}-x_{1 m} x_{3 m}, \\
\dot{x}_{3 m}=x_{1 m} x_{2 m}-p_{3} x_{3 m}
\end{array}\right.
$$

where $p_{1}, p_{2}$ and $p_{3}$ are positive constants which are chosen such that system (37) has a chaotic behaviour. We take system (37) as the master system. The slave system is defined as follows

$$
\left\{\begin{array}{l}
\dot{x}_{1 s}=q_{1}\left(x_{2 s}-x_{1 s}\right)+u, \\
\dot{x}_{2 s}=q_{2} x_{1 s}-x_{2 s}-x_{1 s} x_{3 s}, \\
\dot{x}_{3 s}=x_{1 s} x_{2 s}-q_{3} x_{3 s},
\end{array}\right.
$$

where we have introduced a feedback control law $u$. We have assumed that the parameters $q_{1}, q_{2}$ and $q_{3}$ are different from the parameters of the master system (37). Subtracting equation (37) from equation (38) and using the notation $e_{1}=x_{1 s}-x_{1 m}$, $e_{2}=x_{2 s}-x_{2 m}$ and $e_{3}=x_{3 s}-x_{3 m}$ yields

$$
\left\{\begin{array}{l}
\dot{e}_{1}=\Delta F+u, \\
\dot{e}_{2}=q_{2} e_{1}-e_{2}-e_{1} e_{3}+G_{1}, \\
\dot{e}_{3}=e_{1} e_{2}-q_{3} e_{3}+G_{2},
\end{array}\right.
$$

where

$$
\begin{aligned}
& \Delta F=q_{1}\left(e_{2}-e_{1}\right)+\left(q_{1}-p_{1}\right) x_{2 m}+\left(p_{1}-q_{1}\right) x_{1 m}, \\
& G_{1}=\left(q_{2}-p_{2}\right) x_{1 m}-e_{1} x_{3 m}-e_{3} x_{1 m} \\
& G_{2}=\left(p_{3}-q_{3}\right) x_{3 m}+e_{1} x_{2 m}+e_{2} x_{1 m} .
\end{aligned}
$$


Now, defining the master system output by $y_{m}=x_{1 m}$ and the slave system output by $y_{s}=x_{1 s}$, the error system output is $y=h(e)=e_{1}$. With this in mind, the smallest integer is $\rho=1$. In this way, the coordinates transformation is globally defined by $z_{1}=e_{1}, \zeta_{1}=e_{2}$ and $\zeta_{2}=e_{3}$. Then, the uncertain system (39) can be rewritten as follows:

$$
\left\{\begin{array}{l}
\dot{z}_{1}=\Delta F+u, \\
\dot{\zeta}_{1}=q_{2} z_{1}-\zeta_{1}-z_{1} \zeta_{2}+G_{1}, \\
\dot{\zeta}_{2}=z_{1} \zeta_{1}-q_{3} \dot{\zeta}_{2}+G_{2}, \\
y=z_{1},
\end{array}\right.
$$

where $y$ denotes the output of the error uncertain system. In order to illustrate that system (40) satisfies the minimum phase assumption, one may prove that $\dot{\zeta}_{1}=q_{2} z_{1}-\zeta_{1}-z_{1} \zeta_{2}+G_{1}$ and $\dot{\zeta}_{2}=z_{1} \zeta_{1}-q_{3} \dot{\zeta}_{2}+G_{2}$ converge asymptotically to zero when $z_{1}=0$. Note that $\zeta=\left(\zeta_{1}, \zeta_{2}\right)^{T}$ is uncertain and $\zeta$ is bounded. Thus, the zero dynamics can be written as

$$
\dot{\zeta}=E \zeta+H
$$

where $H=\left(G_{1}, G_{2}\right)^{T}$ and

$$
E=\left[\begin{array}{rr}
-1 & 0 \\
0 & -q_{3}
\end{array}\right],
$$

which is Hurwitz because $q_{3}>0$. Since $G_{1}$ and $G_{2}$ are bounded, the zero dynamics subsystem $\dot{\zeta}=E \zeta+H$ is asymptotically stable. Hence, system (40) is minimum phase. Since Assumptions 1 and 2 are satisfied, the dynamical system (40) can be transformed into its equivalent canonical form (6) (with $\eta=\Delta F$ as the augmented state). So the extended state observer (24) can be described in the following form:

$$
\left\{\begin{array}{l}
\dot{\hat{z}}_{1}=\hat{\eta}-L \lambda_{1}\left|\hat{z}_{1}-z_{1}\right|^{\frac{1}{2}} \operatorname{sgn}\left(\hat{z}_{1}-z_{1}\right)+u, \\
\dot{\hat{\eta}}=-L^{2} \lambda_{2} \operatorname{sgn}\left(\hat{z}_{1}-z_{1}\right) .
\end{array}\right.
$$

Hence, the robust adaptive feedback (28) and (29) can be described as

$$
u(\hat{z})= \begin{cases}0 & t<t_{0}, \\ -\hat{\eta}-\frac{1}{2} B^{\top} P \hat{z}_{1} & t_{0} \leq t \leq T, \\ -\hat{\eta}-\frac{1}{2} B^{\top} \bar{P} \hat{z}_{1} & t>T\end{cases}
$$

In the numerical simulations, the initial conditions of the master and slave systems are respectively $\left(x_{1 m}(0), x_{2 m}(0), x_{3 m}(0)\right)=(8,3,1)$ and $\left(x_{1 s}(0), x_{2 s}(0), x_{3 s}(0)\right)=(10,15,9)$. We choose $\left(\hat{z}_{1}(0), \hat{\eta}(0)\right)=(2,0)$. The master parameter values are the following: $p_{1}=10$, $p_{2}=28, p_{3}=8 / 3$ and the slave parameters values are: $q_{1}=9.95, q_{2}=27.86$ and $q_{3}=2.653$ corresponding to $0.5 \%$ of parameter mismatches. The other parameters are chosen as: $\lambda_{1}=1, \lambda_{2}=5$ and $L=100$. The controller parameters were $Q=1 \times 10^{6}$, 
$Q_{f}=1,000, \bar{P}=1,000$. The controller is turned on at $t_{0}=0$ and the control time (finite horizon) is established at $T=0.04 \mathrm{sec}$.

Figure 5 depicts the simulation results of the synchronisation errors. One can observe that the synchronisation error converge to the origin by using the robust adaptive feedback (41) and (42) in spite of the fact that both master and slave systems have different parameters values. A fairly good convergence of $e_{1}$ is obtained in about $0.04 \mathrm{sec}$ which corresponds to the finite horizon [see Figure 5(b)]. Also, although the control input is acting only on the state $e_{1}$, the other synchronisation errors $e_{2}$ and $e_{3}$ also converge to zero. The performance of the proposed robust adaptive feedback $u(t)$ and synchronisation graphs between slave and master $\left(x_{s}\right.$ versus $\left.x_{m}\right)$ are depicted in Figure 6. Figure 7 investigates the robustness of the proposed scheme in front of noise and parameter mismatch $(0.5 \%)$. It appears that, when $s n r$ increases the synchronisation is better.

In comparison, some additional simulation studies are conducted to show the effect of the controller parameters on the time evolution of synchronisation error norm $\left|e_{1}\right|$ and on the energy wasted by the control action. Figure 8 show the effect of the controller parameters on the time evolution of error norm $\left|e_{1}\right|$ when $Q$ is varied and when $Q_{f}=\bar{P}=\sqrt{Q}$. As the previous example one can see that, when the parameter $Q$ increases the synchronisation is better. Figure 9 shows the time evolution of the control signal $u(t)$ for three different values of parameter $Q$. After a very short transient period one can see that, when the parameter $Q$ increases the amplitude of the controller's signal decreases. Therefore one can conclude that, when the parameter $Q$ increases the energy wasted by the control action decreases.

Figure 5 Numerical simulation results of synchronisation between two Lorenz oscillators, (a) chaotic attractor corresponding to the master system during synchronisation; dynamics of the synchronisation errors with $0.5 \%$ of parameter mismatches (b) $e_{1}=x_{1 s}-x_{1 m}$, (c) $e_{2}=x_{2 s}-x_{2 m}$ and (d) $e_{3}=x_{3 s}-x_{2 m}$

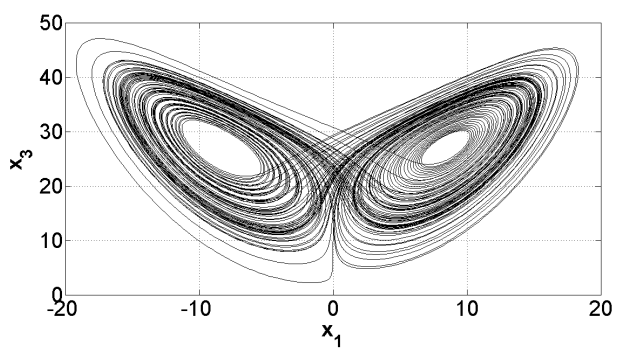

(a)

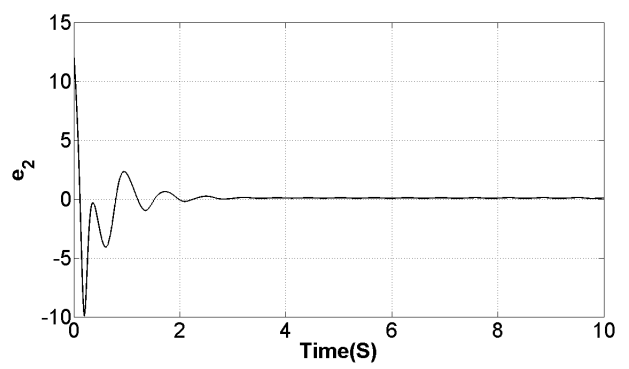

(c)

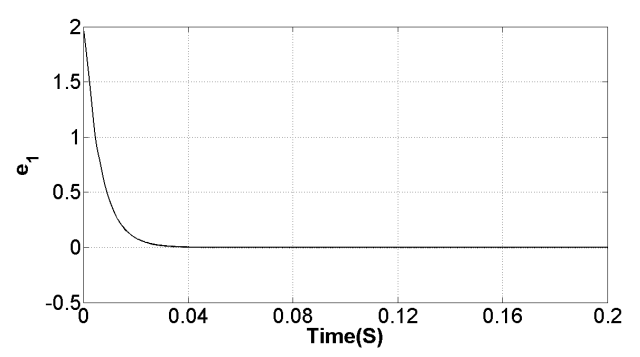

(b)

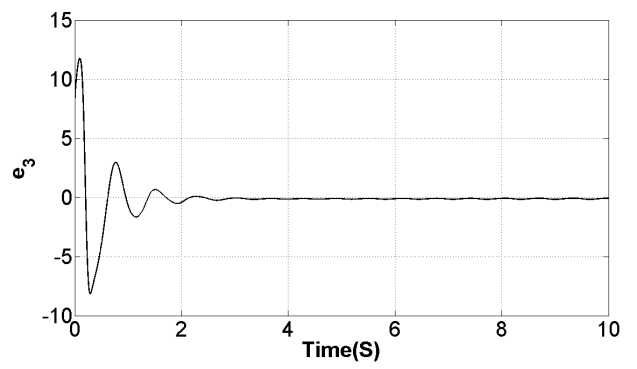

(d) 
Figure 6 Performance of the proposed feedback control $u(t)$, (a) phase portraits of (b) $x_{1 s}$ versus $x_{1 m}$, (c) $x_{2 s}$ versus $x_{2 m}$ and (d) $x_{3 s}$ versus $x_{3 m}$

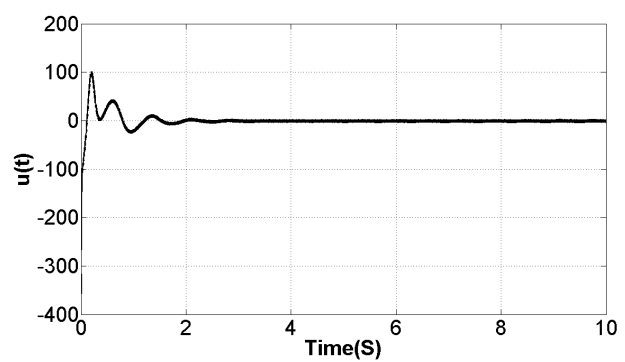

(a)

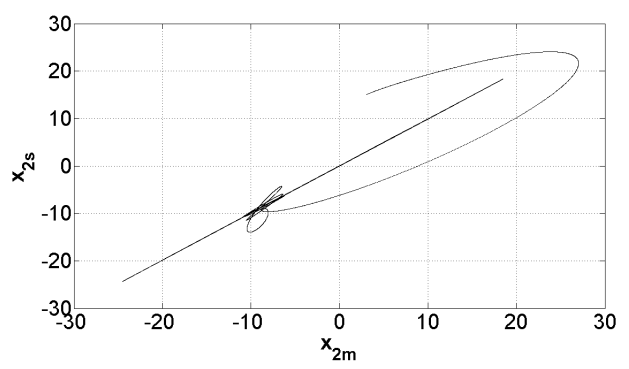

(c)

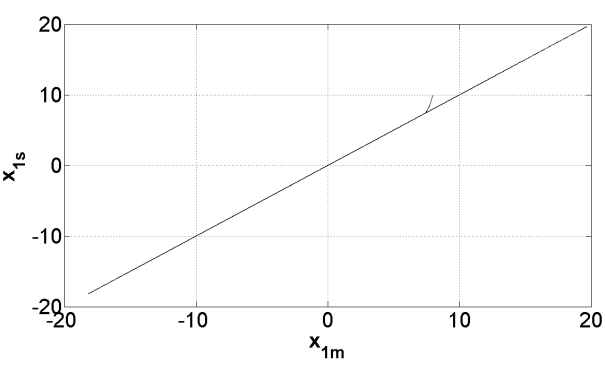

(b)

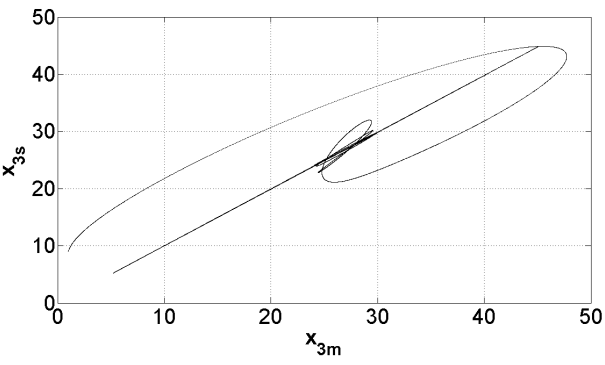

(d)

Figure 7 Time evolution of error norm $\left|e_{1}\right|$ when $s n r$ is varied (see online version for colours)

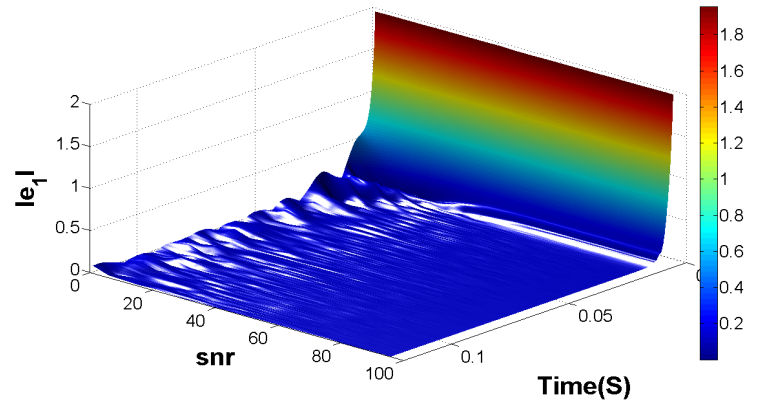

Figure 8 Time evolution of error norm $\left|e_{1}\right|$ when parameter $Q$ is varied (see online version for colours)

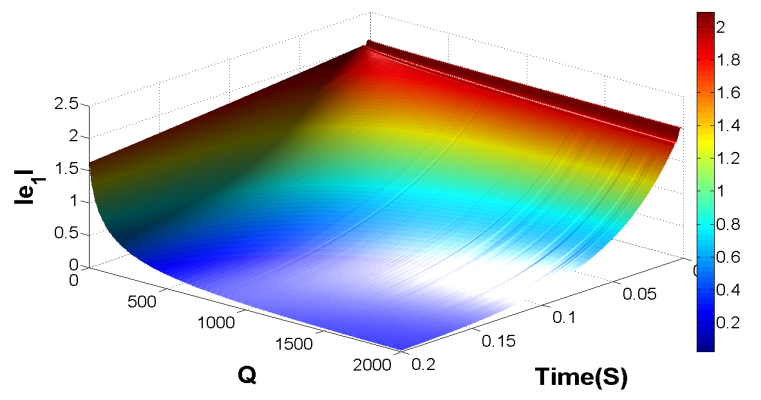


Figure 9 Time evolution of the control signal $u(t)$ for three different values of parameter $Q$ (see online version for colours)

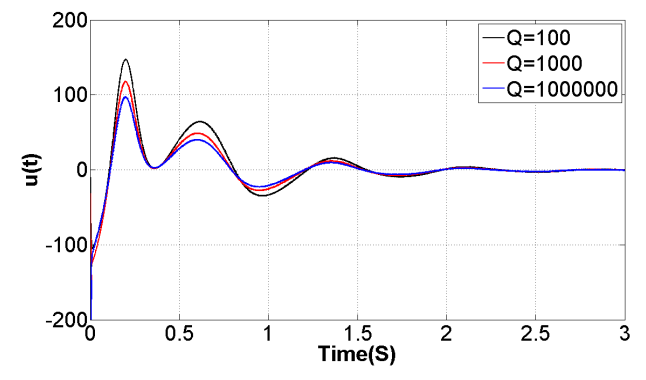

\subsection{Synchronisation of two strictly different systems}

Herein, we study the synchronisation of two strictly different chaotic oscillators, that is, two chaotic systems of the same order which have different models. The goal is to show that the synchronisation can be achieved in spite of model differences between the master and slave systems and external perturbations by oscillatory signals which can be interpreted as noise. In particular, we consider the case where the master system is represented by the extended Van der Pol equation:

$$
\left\{\begin{array}{l}
\dot{x}_{1 m}=x_{2 m} \\
\dot{x}_{2 m}=p_{1}\left(1-x_{1 m}^{2}\right) x_{2 m}-p_{2}^{2} x_{1 m}-p_{3} x_{1 m}^{3}-p_{4} x_{1 m}^{5}+p_{5} \cos w_{1} t
\end{array}\right.
$$

and the slave oscillator is given by the extended Rayleigh equation

$$
\left\{\begin{array}{l}
\dot{x}_{1 s}=x_{2 s}, \\
\dot{x}_{2 s}=q_{1}\left(1-x_{2 s}^{2}\right) x_{2 s}-q_{2} x_{1 s}-q_{3} x_{1 s}^{3}-q_{4} x_{1 s}^{5}+q_{5} \cos w_{2} t+u,
\end{array}\right.
$$

where $p_{i}, q_{i}$ and $w_{i}, i=1,2$ are constants and $u$ is the control force to be chosen. If $y_{m}=h\left(x_{m}\right)=x_{1 m}$ and $y_{s}=h\left(x_{s}\right)=x_{1 s}$ are the outputs of both master and slave oscillators, then one has $y=h(e)=e_{1}$. Now, let us define $e \in \mathbb{R}^{2}$ by $e_{i}=x_{i s}-x_{i m}, i=1,2$ ( $e$ is a vector whose components represent the synchronisation error). Then, the resulting error dynamical system can be expressed as

$$
\left\{\begin{array}{l}
\dot{e}_{1}=e_{2}, \\
\dot{e}_{2}=\Delta F\left(e, x_{m}, t\right)+u \\
y=e_{1}
\end{array}\right.
$$

where $y$ is the output of the uncertain system and

$$
\begin{aligned}
\Delta F\left(e, x_{m}, t\right)= & q_{1}\left(1-x_{2 s}^{2}\right) x_{2 s}-q_{2} x_{1 s}-q_{3} x_{1 s}^{3}-q_{4} x_{1 s}^{5}+q_{5} \cos w_{2} t \\
& -p_{1}\left(1-x_{1 m}^{2}\right) x_{2 m}+p_{2}^{2} x_{1 m}+p_{3} x_{1 m}^{3}+p_{4} x_{1 m}^{5}-p_{5} \cos w_{1} t
\end{aligned}
$$


contains the two system's model difference, which is unknown to us. Thus, the coordinates transformation is given by $z_{1}=e_{1}$ and $z_{2}=e_{2}$. In such a way, system (45) is transformed into

$$
\left\{\begin{array}{l}
\dot{z}_{1}=z_{2}, \\
\dot{z}_{2}=\Theta\left(z, x_{m}, t\right)+u,
\end{array}\right.
$$

where $\Theta\left(z, x_{m}, t\right)=\Delta F\left(z, x_{m}, t\right)$. Note that system (46) is fully linearisable, i.e., there is no unobservable state $\zeta$ in the uncertain system (46) because the relative degree is $\rho=n=2$. Now, defining $\eta=\Theta\left(z, x_{m}, t\right)$, system (46) can be transformed into its equivalent form (6). Then, we get the extended state observer (24) as the following form:

$$
\left\{\begin{array}{l}
\dot{\hat{z}}_{1}=\hat{z}_{2}-L \lambda_{1}\left|\hat{z}_{1}-z_{1}\right|^{2 / 3} \operatorname{sgn}\left(\hat{z}_{1}-z_{1}\right), \\
\dot{\hat{z}}_{2}=\hat{\eta}-L^{2} \lambda_{2}\left|\hat{z}_{1}-z_{1}\right|^{1 / 2} \operatorname{sgn}\left(\hat{z}_{1}-z_{1}\right)+u, \\
\dot{\hat{\eta}}=-L^{3} \lambda_{3} \operatorname{sgn}\left(\hat{z}_{1}-z_{1}\right) .
\end{array}\right.
$$

So, the robust adaptive feedback (28) and (29) can be described as

$$
u(\hat{z})= \begin{cases}0 & t<t_{0}, \\ -\hat{\eta}-\frac{1}{2} B^{\top} P \hat{z}_{1} & t_{0} \leq t \leq T, \\ -\hat{\eta}-\frac{1}{2} B^{\top} \bar{P} \hat{z}_{1} & t>T\end{cases}
$$

Figure 10 Phase portraits of the extended Van der Pol and Rayleigh oscillators without the feedback coupling, (a) attractor of the extended Van der Pol oscillator and (b) attractor of the extended Rayleigh equation

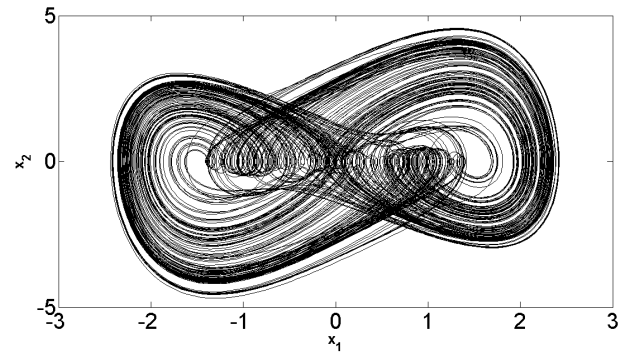

(a)

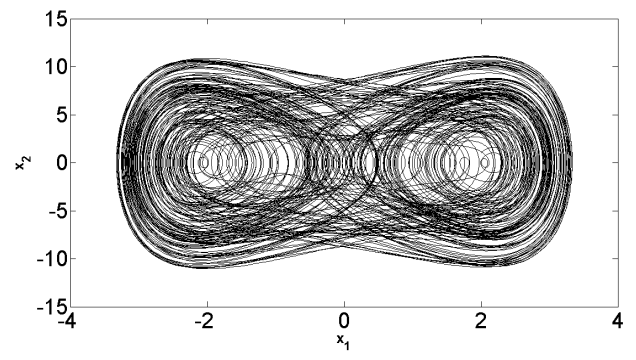

(b)

In numerical simulations, the parameter values for the extended Van der Pol oscillator were chosen as $p_{1}=0.4, p_{2}=0.46, p_{3}=1, p_{4}=0.1, p_{5}=4.5$ and $w_{1}=0.86$ such that system (43) exhibits a chaotic behaviour. Figure 10(a) shows the phase portrait of the extended Van der Pol oscillator. For the parameter values $q_{1}=0.001, q_{2}=1, q_{3}=0.8$, $q_{4}=0.3, q_{5}=26$ and $w_{2}=1$, the extended Rayleigh equation displays chaotic behaviour when $u=0$. Figure $10(\mathrm{~b})$ presents the phase portrait of the extended Rayleigh equation for $u=0$ for all $t \geq 0$ (uncontrolled evolution). The initial conditions were chosen as follows: $\left(x_{1 m}(0), x_{2 m}(0)\right)=(1,0)$ and $\left(x_{1 s}(0), x_{2 s}(0)\right)=(2,2)$. The attractors of the two systems are not the same, as one might expect as the systems are different. The initial 
conditions of the observer (47) were chosen as follows: $\left(\hat{z}_{1}(0), \hat{z}_{2}(0), \hat{\eta}(0)\right)=(1,2,0)$. We choose $\lambda_{1}=10, \lambda_{2}=5, \lambda_{3}=5$ and $L=3$. The controller is turned on at $t_{0}=0$ with a finite horizon at $T=5 \mathrm{sec}$. The weighting matrices are given by

$$
Q=\left[\begin{array}{cc}
2,500 & 0 \\
0 & 2,500
\end{array}\right], \quad Q_{f}=\left[\begin{array}{cc}
50 & 0 \\
0 & 50
\end{array}\right],
$$

and the corresponding tuning parameter for $t>T$ is

$$
\bar{P}=\left[\begin{array}{cc}
50 & 50 \\
50 & 50
\end{array}\right] \text {. }
$$

Figure 11 Dynamics of the synchronisation errors, (a) $e_{1}=x_{1 s}-x_{1 m}$ and (b) $e_{2}=x_{2 s}-x_{2 m}$

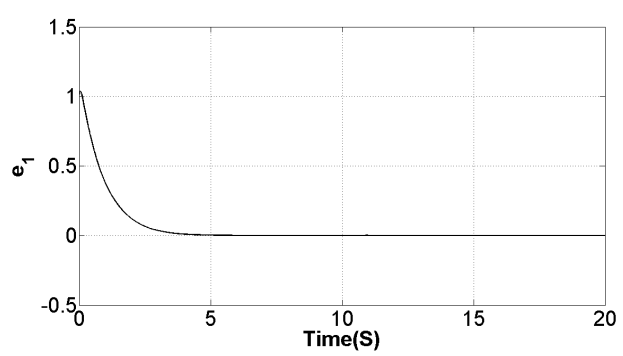

(a)

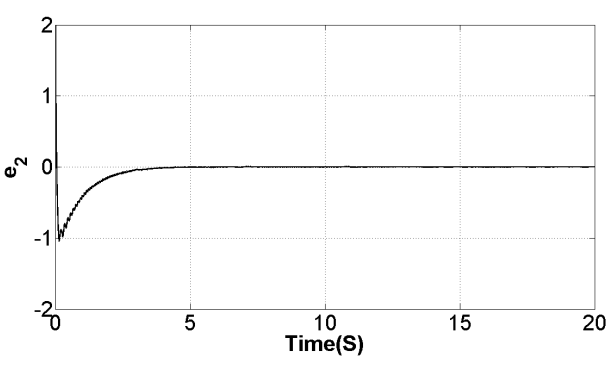

(b)

Figure 12 Complete exact synchronisation of the extended Rayleigh equation and the extended Van der Pol oscillator via the robust adaptive feedback (47) and (48), (a, b) states of the drive system are tracked by states of the response system $(c, d)$ phase diagram of $x_{1 s}$ versus $x_{1 m}$ and $x_{2 s}$ versus $x_{2 m}$

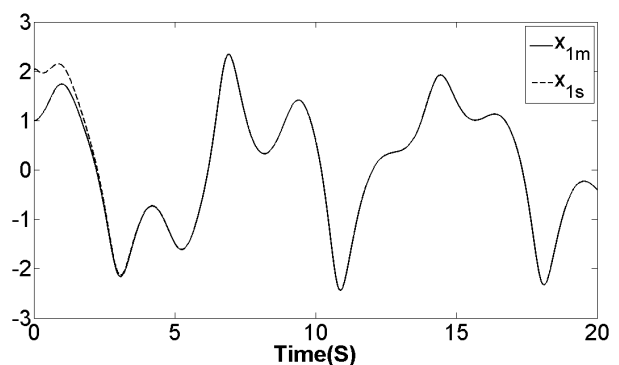

(a)

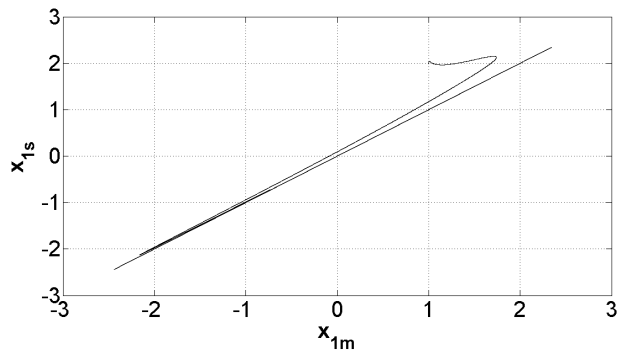

(c)

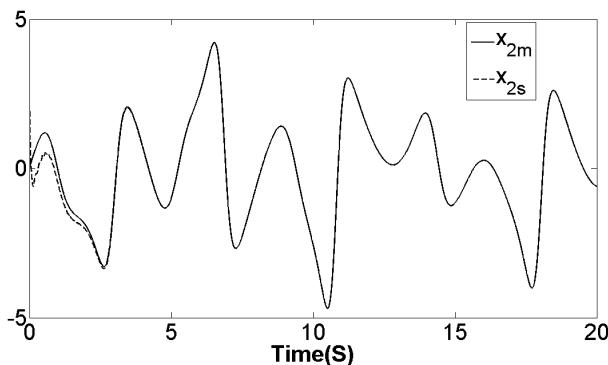

(b)

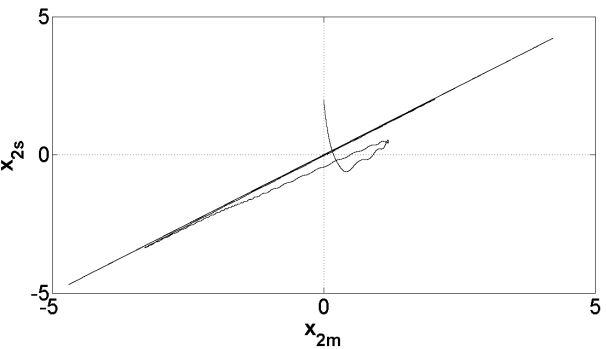

(d) 
Figure 11 present the time evolution of the synchronisation errors. One can see that the synchronisation error are stabilised at the origin in spite of strictly different models. In this way, the robust adaptive feedback (47) and (48) yields a complete synchronisation of the chaotic oscillators in the sense that the trajectories of the discrepancy error system converge exactly to the origin, it appears that the synchronisation of the drive and response systems is attained in about $5 \mathrm{sec}$ which corresponds to the finite horizon. Figures 12(a) and 12(b) shows the dynamical evolution of the extended Rayleigh and extended Van der Pol oscillators. It is found that the states of the slave system are tracked by the states of the master states. In order to add evidence, we have plotted $x_{1 m}$ versus $x_{2 m}$ and $x_{1 s}$ versus $x_{2 s}$ in Figures 12(c) and 12(d). It illustrates that the manifolds $x_{1 s}=x_{1 m}$ and $x_{2 s}=x_{2 m}$ are stable and the synchronisation objective is attained.

\section{Conclusions}

In this paper a novel robust control scheme using the Ricatti equations for synchronising a class of chaotic systems with uncertainties is proposed. The main idea is to construct an augmented dynamical system from the synchronisation error system, which is itself uncertain. We propose a robust feedback control that takes into account the behaviour of transient response and the feedback effort (i.e., the energy wasted by the control action). Thus, the proposed strategy allows to set specifically the time horizon for the synchronisation of chaotic systems. To illustrate the effectiveness of the design, the synchronisation of two Chua's oscillators, two Lorenz oscillators, and extended Van der Pol and Rayleigh equations were used as illustrative examples. Both theoretical and simulation results reveal the validity of the proposed control technique for synchronising uncertain chaotic oscillators. As future work, we will try to implement physically this proposed strategy.

\section{Acknowledgements}

The authors would like to express their sincere gratitude to editor for handling the process of reviewing the paper, as well as to the anonymous reviewers who carefully reviewed the manuscript.

\section{References}

Aguilar, R., Martinez-Guerra, R., Poznyak, A. and Maya-Yescas, R. (2002) 'Temperature control in catalytic cracking reactors via robust PID controller', Journal of Process Control, Vol. 12, No. 6, pp.695-705.

Aguilar-Lopez, R. and Alvarez-Ramirez, J. (2002) 'Sliding-mode control scheme for a class of continuous chemical reactors', IEEE Proceedings of Control Theory and Applications, Vol. 149, No. 4, pp.263-268.

Anderson, B.D.O. and Moor, J.B. (1990) Optimal Control. Linear Quadratic Methods, Prentice Hall, New York.

Bowong, S., Moukam Kakmeni, F.M. and Koina, R. (2006) 'Chaos synchronization and duration time of a class of uncertain chaotic sys tem', Mathematics and Computers in Simulation, Vol. 71, No. 3, pp.212-228. 
Chembo, Y. and Woafo, P. (2002) 'Stability and optimization of chaos synchronization through feedback coupling with delay', Physics Letters A, Vol. 298, No. 1, pp.18-28.

Chen, G. and Dong, X. (1998) Form Chaos to Order. Methodologies, Perspectives and Applications, World Scientific, Singapore.

Chen, Y., Chen, X. and Gu, S. (2007) 'Lag synchronization of structurally nonequivalent chaotic systems with time delays', Nonlinear Analysis, Vol. 66, No. 9, pp.1929-1937.

Femat, R. (2002) 'An extension to chaos control via lie derivative: fully linearizable systems', Chaos, Vol. 12, No. 4, pp.1027-1033.

Femat, R. and Solís-Perales, G. (2008) Robust Synchronization of Chaotic Systems via Feedback, Springer.

Femat, R., Alvarez-Ramírez, J. and Fernandez-Anaya, G. (2000) 'Adaptive synchronization of high-order chaotic systems: a feedback with low-order parametrization', Physica D, Vol. 139, Nos. 3-4, pp.231-246.

Femat, R., Alvarez-Ramírez, J., Castillo-Toledo, B. and Gonz'ales, J. (1999) 'On robust chaos suppression in a class of non driven oscillators: application to the Chua's circuit', IEEE Transactions on Circuits and Systems, Part I, Vol. 46, No. 9, pp.1150-1152.

Femat, R., Jimenez, C., Bowong, S. and Solís-Perales, G. (2009) 'Accounting the control effort to improve chaos suppression via robust adaptive feedback', International Journal Modelling, Identification and Control, Vol. 6, No. 2, pp.147-155.

Han, J.Q. (1995) 'The extended state observer of a class of uncertain systems', Control and Decision, Vol. 10, No. 1, pp.85-88.

Hu, J., Chen, S. and Chen, L. (2005) 'Adaptive control for anti-synchronization of Chua's chaotic system', Physics Letters A, Vol. 339, No. 6, pp.455-460.

Isidori, A. (1989) Nonlinear Control Systems, Springer-Verlag, Berlin, Germany.

Kammogne, S.T. and Fotsin, H.B. (2014) 'Adaptive control for modified projective synchronization-based approach for estimating all parameters of a class of uncertain systems: case of modified colpitts oscillators', Journal of Chaos, Vol. 2014, Article ID 659647, pp.1-13 [online] http://dx.doi.org/10.1155/2014/659647 (accessed 7 December 2015).

Keel, L.H. and Bhattacharyya, S.P. (1997) 'Robust, fragile or optimal', IEEE Transactions on Automatic Control, Vol. 42, No. 8, pp.1098-1105.

Keel, L.H. and Bhattacharyya, S.P. (1998) 'Author's reply', IEEE Transactions on Automatic Control, Vol. 43, No. 9, pp.1268-1272.

Kocarev, L., Parlitz, U. and Hu, B. (1998) 'Lie derivatives and dynamical systems', Chaos Solitons \& Fractals, Vol. 9, No. 8, pp.1359-1366.

Li, G.H. (2005) 'Synchronization and anti-synchronization of Colpitts oscillators using active control', Chaos Solitons \& Fractals, Vol. 26, No. 1, pp.87-93.

Li, G.H. and Zhou, S.P. (2006) 'An observer-based anti-synchronization', Chaos Solitons \& Fractals, Vol. 29, No. 2, pp.495-498.

Li, X.R., Zhao, L.Y. and Zhao, G.Z. (2005) 'Sliding mode control for synchronization of chaotic systems with structure or parameters mismatching', Journal of Zhejiang University, Vol. 6, No. 6, pp.571-576.

Mäkilä, P.M. (1998) 'Comments on robust, fragile or optimal', IEEE Transactions on Automatic Control, Vol. 43, No. 9, pp.1265-1267.

Mao, Y., Tang, K.S., Liu, Y. and Kocarev, L. (2009) 'Identification of biological neurons using adaptive observers', Cognitive Processing, Vol. 10, 1 Suppl., pp.41-53.

Mbe, E.S.K., Fotsin, H.B., Kengne, J. and Woafo, P. (2014) 'Parameters estimation based adaptive Generalized Projective Synchronization (GPS) of chaotic Chua's circuit with application to chaos communication by parametric modulation', Chaos Solitons \& Fractals, Vol. 61, pp.27-37, DOI: 10.1016/j.chaos.2014.02.004.

Michael, G.R., Arkady, S.P. and Kurths, J. (1996) 'Phase synchronization of chaotic oscillators', Physical Review Letters, Vol. 76, No. 11, pp.1804-1807. 
Murali, K. and Lakshmanan, M. (1998) 'Secure communication using a compound signal from generalized synchronizable chaotic systems', Physics Letters A, Vol. 241, No. 6, pp.303-310.

Osipov, G.V., Pikovsky, A.S., Rosemblum, M.G. and Kurths, J. (1997) 'Phase synchronization effects in a lattice of nonidentical Rossler oscillators', Physical Review E, Vol. 55, No. 3, pp.2353-2361.

Pecora, L.M. and Caroll, T.L. (1990) 'Synchronization in chaotic systems', Physical Review Letters, Vol. 64, No. 8, pp.821-834.

Pyragas, K. (1998) 'Synchronization of coupled time-delay systems: analytical estimations', Physical Review E, Vol. 58, No. 3, pp.3067-3071.

Santoboni, G., Pogromsky, A.Y. and Nijmeijer, H. (2001) 'An observer for phase synchronization of chaos', Physics Letters A, Vol. 291, Nos. 4-5, pp.265-273.

Taherion, I.S. and Lai, Y.C. (1999) 'Observability of lag synchronization of coupled chaotic oscillators', Physical Review E, Vol. 59, No. 6, pp.R6247-R6250.

Wang, Y.W. and Guan, Z.H. (2006) 'Generalized synchronization of continuous chaotic systems', Chaos Solitons \& Fractals, Vol. 27, No. 1, pp.97-101.

Woafo, P. and Kreankel, R. (2002) 'Synchronization: Stability and duration time', Physical Review E, Vol. 65, No. 3, pp.036225-036231.

Yang, S.S. and Duan, C.K. (1998) 'Generalized synchronization in chaotic systems', Chaos Solitons \& Fractals, Vol. 9, No. 10, pp.1703-1707. 